\title{
Solitary Brain Metastasis Detected 1 Year After Robot- Assisted Laparoscopic Radical Nephrectomy: A Case Report
}

\author{
Ji Kang Yun', Woong Bin Kim¹, Si Hyun Kim², Ahrim Moon³, Sang Wook Lee ${ }^{1}$ \\ 'Department of Urology, Soonchunhyang University Bucheon Hospital, Bucheon, Korea \\ ${ }^{2}$ Department of Urology, Soonchunhyang University Cheonan Hospital, Cheonan, Korea \\ ${ }^{3}$ Department of Pathology, Soonchunhyang University Bucheon Hospital, Bucheon, Korea
}

Brain metastases are not rare in patients with renal cell carcinoma. However, there have been few reports of isolated brain metastases. In patients with localized renal masses, urologists tend to evaluate only the chest and abdomen region. We report the case of a 65 -year-old patient who presented with sudden left-sided weakness. He underwent brain surgery after a mass was found on brain imaging. Histopathology analysis revealed a single brain metastasis that occurred 15 months after robot-assisted laparoscopic radical nephrectomy for renal cell carcinoma. Although the patient had been evaluated for localized kidney cancer in preoperative staging, he had not complained of any neurological symptoms before or after radical nephrectomy. Therefore, we had not perceived a need for brain evaluation at that time. This report is intended to draw attention to the need for further evaluation in patients with large renal masses, as well as patients with moderate to high recurrence risk scores.

Key Words: Renal cell carcinoma, Neurologic symptoms, Brain tumor, Brain metastases
Received October 18, 2021

Accepted November 22, 2021

Corresponding author:

Sang Wook Lee

Email: bartol@schmc.ac.kr

https://orcid.org/0000-0001-9660-4092
Renal cell carcinoma (RCC) comprises approximately $2 \%$ of cancers and causes approximately $2 \%$ of cancer-related deaths worldwide. RCC caused approximately 14,000 deaths in 2016 in the United States. ${ }^{1}$ RCC has a lifetime prevalence of approximately 1.34\%; furthermore, approximately $30 \%$ of RCC patients exhibit a metastasis at the time of diagnosis. ${ }^{2}$ Patients with RCCs confined to the kidneys are treated by partial or radical nephrectomy. Recurrence occurs in approximately $27 \%$ of affected patients after surgery. ${ }^{3}$ The most common histological type of RCC is clear cell, which comprises $75 \%-80 \%$ of cases. RCCs commonly metastasize through a hematogenous route. The most common metastatic sites in clear- cell RCCs are lungs, lymph nodes, bone, and liver. Brain involvement reportedly ranges from 3.9\% to $24 \% .{ }^{4}$ RCCs are less likely to metastasize to the brain, compared with other sites; the mean interval between nephrectomy and brain metastasis is $1-3$ years. A few cases of brain metastasis occurring 15-20 years after nephrectomy in RCC patients have been reported., ${ }^{5,6}$ Distant brain metastases are generally multiple. The prognoses for patients with single or multiple RCC brain metastases are poor and mortality rates are high. RCC brain metastases are associated with substantial peritumoral edema; they may cause intracranial hemorrhage because of extensive vascularity. Here, we describe a patient who exhibited single brain metastasis at 15 
months after robot-assisted laparoscopic radical nephrectomy for RCC. Although the patient had been evaluated for localized kidney cancer in preoperative staging, he did not complain of any neurological symptoms before or after surgery.

\section{CASE REPORT}

A 65-year-old man presented to the Emergency Department with sudden left side weakness and a mild headache. There were no other symptoms, such as flank or abdominal pain, nausea, and vomiting. The patient had a medical history of chronic obstructive pulmonary disease and asthma. He had no history of coronary artery disease or cerebrovascular accident, and there was no family history of brain disease. Complete blood count, blood urea nitrogen, creatinine, electrolytes, and liver function test findings were within normal ranges. On physical examination, vital signs were stable. The patient had decreased motor and sensory responses in the left upper and lower limbs, compared with the right side. Dysphagia was observed but orientation was not impaired. The patient had previously undergone a robot-assisted laparoscopic radical nephrectomy for RCC, approximately 15 months prior (Fig. 1). The tumor size was $9.5 \mathrm{~cm} \times 7.0 \mathrm{~cm} \times 6.5 \mathrm{~cm}$. The tumor extended up to renal sinus fat but not beyond Gerota's fascia. The pathological stage was T3a according to the 8th edition of the cancer staging system established by the American Joint Committee on Cancer. The final histopathological diagnosis was clear-cell type RCC with Fuhrman nuclear grade 4 . Resection margins were negative. Immunohistochemical analysis revealed that the tumor tissue was positive for CD10 and negative for cytokeratin 7 (Fig. 2). The patient was discharged on the fifth postoperative day without any complications. Follow-up abdominal and chest computed tomography (CT) scans were performed at 6-month intervals. Preoperatively, the patient had a 3-mm intrapulmonary lymph node or inflammatory nodule in the left upper lung on chest CT, which could not be excluded as a metastatic nodule. However, the nodule disappeared spontaneously during the follow-up period (Fig. 3). There was no evidence of metastasis until the patient presented to the Emergency Department with neurological symptoms. Brain CT and magnetic resonance imaging (MRI) revealed a $2.5-\mathrm{cm}$ high-attenuated mass in the right temporal lobe with perilesional edema (Fig. 4). Navigation-guided surgery was performed by the neurosurgery department to remove the mass that was causing the symptoms. Histological analysis of the specimen revealed findings identical to the original RCC. Resection margins were negative. Immunohistochemical analysis revealed that the tumor tissue was positive for CD10 and PAX-8 (Fig. 5). After surgery, whole-
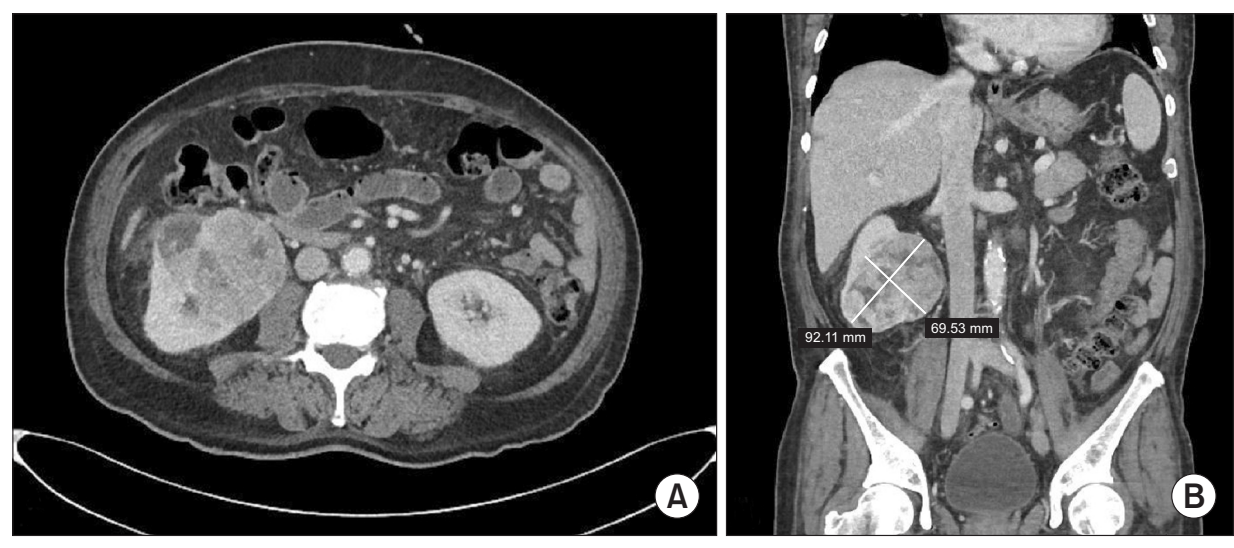

Fig. 1. Computed tomography (CT) scan before radical nephrectomy. Preoperative CT showed a large enhancing mass with perinephric infiltration. Ball-type lesion causing smooth contour bulge, showing heterogenous enhancement of the cystic portion, most likely renal cell carcinoma. No direct invasion was identified. (A) Axial section. (B) Coronal section. 

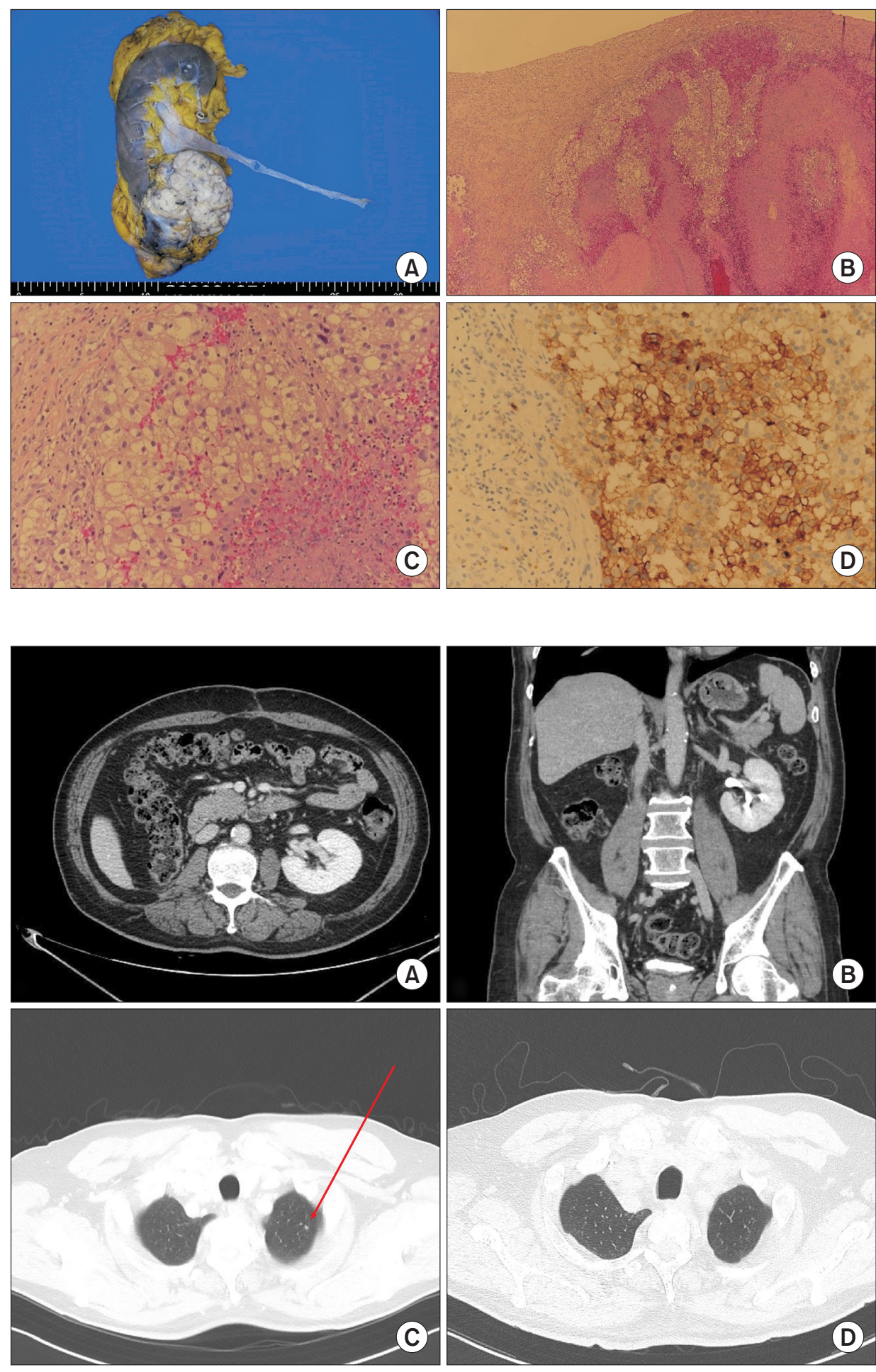

Fig. 2. Pathologic findings. (A) The resected tumor, $9.5 \mathrm{~cm} \times 7.0$ $\mathrm{cm} \times 6.5 \mathrm{~cm}$ in size. (B) The tumor exhibited solid sheet architecture. Central necrosis was also evident on hematoxylin and eosin $(\mathrm{H} \& \mathrm{E})$ staining $(\times 40$ magnification). (C) Tumor cells exhibited abundant cytoplasm that was clear and vacuolated (H\&E: $\times 200$ magnification). (D) Immunohistochemical analysis revealed that the tumor was positive for CD10.
Fig. 3. Computed tomography (CT) after radical nephrectomy. Postoperative CT performed 6 months after surgery showed no abnormal enhancing lesion (right radical nephrectomy state). (A) Axial section. (B) Coronal section. (C) Six-month postoperative chest CT showed a small illdefined nodule in the left upper lobe (i.e., in the apicoposterior segment of the lung), which could not be excluded as a metastatic nodule (arrow). (D) By 12 months postoperatively, the nodule had disappeared spontaneously. body positron emission tomography/CT (PETCT) was performed for RCC restaging workup; no evidence of metastasis was found elsewhere. One month after the surgery, the patient underwent adjuvant chemotherapy with pazopanib, a tyrosine kinase inhibitor. Follow-up brain CT and MRI scans were performed 3 months after surgery. Postoperative hemorrhagic fluid collection and 

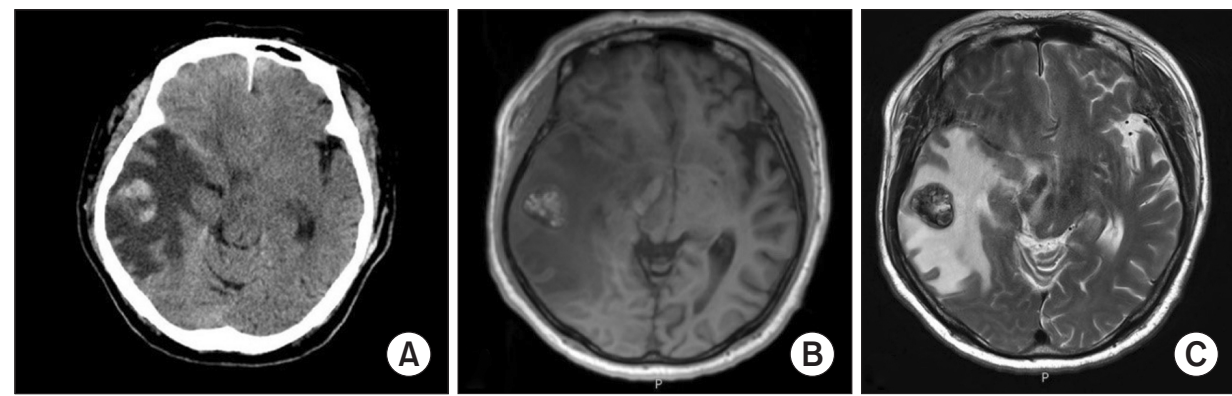

Fig. 4. Preoperative brain imaging. (A) Preoperative computed tomography revealed a high-attenuated mass with perilesional low-attenuated lesion in the right frontal-temporal-parietal lobes, basal ganglia, and right midbrain. (B) T1-weighted magnetic resonance imaging (MRI) revealed a $2.5 \mathrm{~cm}$ heterogeneously enhancing lesion showing severe perilesional edema in the right frontal-temporal-parietal lobes, basal ganglia, and right midbrain. (C) T2-weighted MRI revealed a low-attenuated mass with high-attenuated perilesional edema.
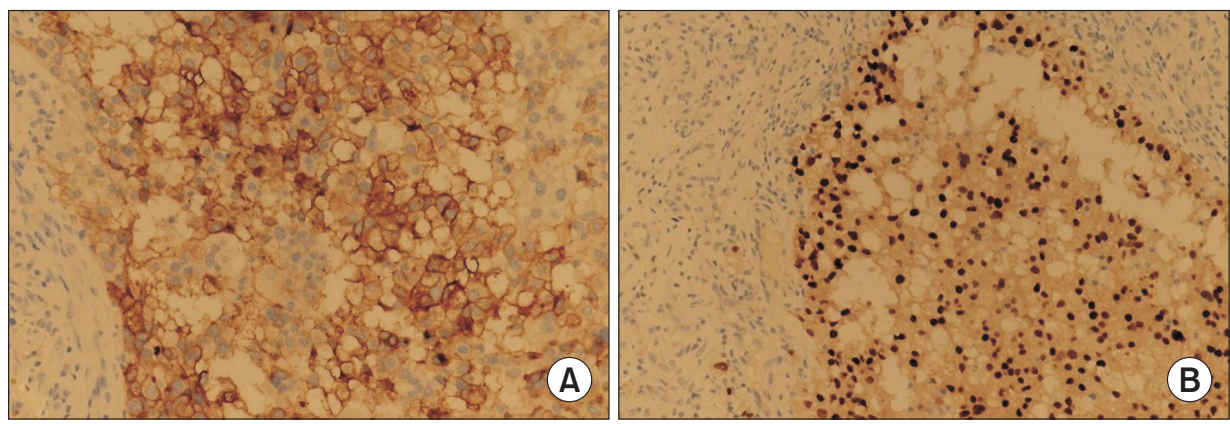

Fig. 5. Immunohistochemical staining of the metastatic brain lesion. (A) CD10 immunohistochemistry showed positive cytoplasmic expression. (B) PAX8 immunohistochemistry showed positive nuclear expression.
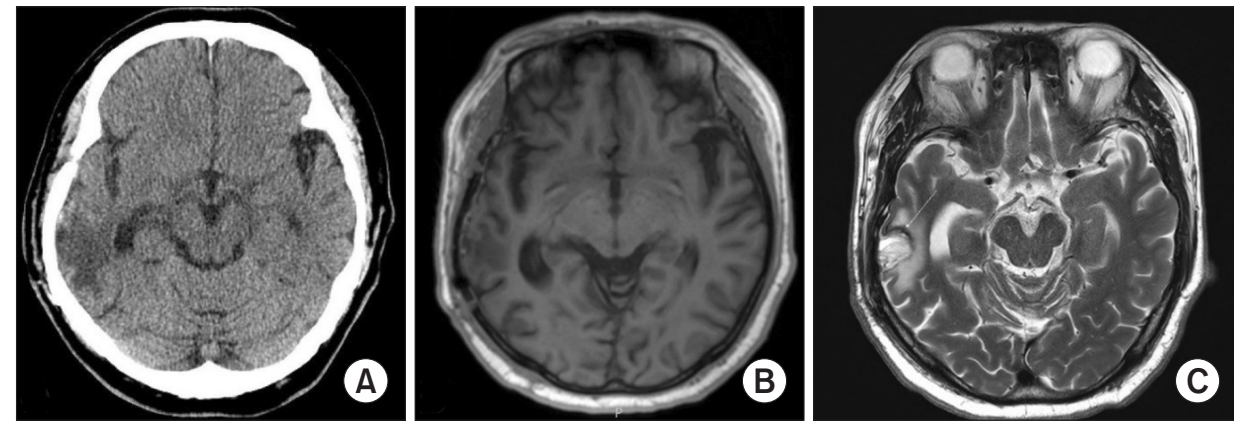

Fig. 6. Postoperative brain imaging. (A) Postoperative brain computed tomography performed 3 months after surgery showed almost completely resolved postoperative hemorrhagic fluid collection and decreased perilesional edema. T1-weighted magnetic resonance imaging (MRI). (B) and T2-weighted MRI. (C) showed slightly decreased irregular, enhancing thickening along the superior-anterior aspect of the right temporal area.

perilesional edema were almost completely resolved (Fig. 6). After 6 months, the patient had no neurological symptoms and there was no further evidence of metastasis on imaging.

Informed written consent was obtained from the patient for publication of this report and any accompanying images.

\section{DISCUSSION}

Brain metastases from RCC occur in approximately $3.9 \%-24 \%$ of affected patients; the mean 
interval between nephrectomy and brain metastasis is $1-3$ years. ${ }^{4,7}$ The prognoses for patients with single or multiple RCC brain metastases are poor and mortality rates are high. The mean survival time after diagnosis is less than 1 year, and the median survival time is $3-4$ months without treatment. $^{8}$

Some current systems may help to estimate the survival rates in RCC patients. The University of California, Los Angeles Integrated Scoring System stratifies patients into 3 risk categories based on pathological stage, nuclear grade, and Eastern Cooperative Oncology Group performance status. The stage, size, grade, and necrosis risk score uses tumor stage, size $(>5 \mathrm{~cm})$, nuclear grade, and tumor necrosis to determine a risk score associated with recurrence in clear-cell RCC patients. ${ }^{10}$ In our patient, the mass was larger than $7 \mathrm{~cm}$, extended to the renal sinus (T3a), and had a Fuhrman nuclear grade of 4. The Eastern Cooperative Oncology Group performance score was 0 . The patient was classified as intermediate risk using the University of California, Los Angeles Integrated Scoring System.

Isolated RCC brain metastases are rare. In most patients, RCC involves adjacent organs first (e.g., lungs, regional lymph nodes, bone, and liver). RCC is also more likely to cause multiple metastases. The 2021 National Comprehensive Cancer Network guidelines recommend performing abdominalpelvic CT or MRI, as well as chest radiography. However, bone scans, chest CT scans, and brain MRI scans are recommended if clinically indicated. The cost-effectiveness of these investigations has not been established thus far. Therefore, screening tests are not routinely performed.

The main treatment options for RCC brain metastases include local aggressive treatments, such as complete surgical resection, stereotactic radiosurgery, or a combination. Complete surgical resection is the treatment of choice in patients with surgically accessible tumors. Surgery provides the best outcomes because it can confirm pathological findings and provide immediate symptomatic relief. It is important to differentiate between these conditions to achieve better survival outcomes. In our patient, the brain mass was completely resected, and the diagnosis was histopathologically confirmed, providing further guidance for treatment decisions.

We hypothesized that the patient already had a brain metastasis at the time of initial diagnosis, but it remained undetected because brain-related screening tests were not performed. Alternatively, the patient might have developed late hematogenous recurrence after surgery. In either situation, it would be meaningful to perform additional tests (e.g., CT scans, MRI scans, bone scans, and PET-CT scans) in patients with a high pathologic T-stage or a high nuclear grade, regardless of symptoms.

In conclusion, during RCC follow-up, careful review of the patient's history and physical examination are important. As demonstrated in this report, patients with large renal masses or intermediate to high recurrence risk scores may benefit from additional investigations, regardless of symptoms. Further well-designed studies are needed to establish cost-effectiveness of routine brain investigations.

\section{NOTES}

- Conflicts of interest: No potential conflict of interest relevant to this article was reported.

- Funding: This work was supported by the Soonchunhyang University Research Fund.

- ORCID

Ji Kang Yun: https://orcid.org/0000-0002-8655-2484

Woong Bin Kim: https://orcid.org/0000-0002-7369-490X

Si Hyun Kim: https://orcid.org/0000-0002-5984-9485

Ahrim Moon: https://orcid.org/0000-0001-6587-0013 
Sang Wook Lee: https://orcid.org/0000-0001-9660-4092

\section{REFERENCES}

1. Bray F, Ferlay J, Soerjomataram I, Siegel RL, Torre LA, Jemal A. Global cancer statistics 2018: GLOBOCAN estimates of incidence and mortality worldwide for 36 cancers in 185 countries. CA Cancer J Clin 2018;68:394-424.

2. Padala SA, Barsouk A, Thandra KC, Saginala K, Mohammed A, Vakiti A, et al. Epidemiology of renal cell carcinoma. World J Oncol 2020;11:79-87.

3. Chin AI, Lam JS, Figlin RA, Belldegrun AS. Surveillance strategies for renal cell carcinoma patients following nephrectomy. Rev Urol 2006;8:1-7.

4. Dudani S, de Velasco G, Wells C, Gan CL, Donskov F, Porta C, et al. Sites of metastasis and survival in metastatic renal cell carcinoma (mRCC): results from the International mRCC Database Consortium (IMDC). J Clin Oncol 2020;38(6_suppl):642.

5. Heckl S, Braun K, Debus J, Kunze S. Cerebral metastasis after primary renal cell carcinoma. J Neurol
Neurosurg Psychiatry 2003;74:141.

6. Bradham RR, Wannamaker CC, Pratt-Thomas HR. Renal cell carcinoma metastases 25 years after nephrectomy. JAMA 1973;223:921-2.

7. Choi WH, Koh YC, Song SW, Roh HG, Lim SD. Extremely delayed brain metastasis from renal cell carcinoma. Brain Tumor Res Treat 2013;1:99-102.

8. Gupta K, Miller JD, Li JZ, Russell MW, Charbonneau C. Epidemiologic and socioeconomic burden of metastatic renal cell carcinoma (mRCC): a literature review. Cancer Treat Rev 2008;34:193-205.

9. Zisman A, Pantuck AJ, Wieder J, Chao DH, Dorey F, Said JW, et al. Risk group assessment and clinical outcome algorithm to predict the natural history of patients with surgically resected renal cell carcinoma. J Clin Oncol 2002;20:4559-66.

10. Zigeuner R, Hutterer G, Chromecki T, Imamovic A, Kampel-Kettner K, Rehak P, et al. External validation of the Mayo Clinic stage, size, grade, and necrosis (SSIGN) score for clear-cell renal cell carcinoma in a single European centre applying routine pathology. Eur Urol 2010;57:102-9. 